\title{
BMJ Open Validating injury burden estimates using population birth cohorts and longitudinal cohort studies of injury outcomes: the VIBES-Junior study protocol
}

\author{
Belinda J Gabbe, ${ }^{1,2,3}$ Joanna F Dipnall, ${ }^{1,4}$ John W Lynch, ${ }^{5,6}$ Frederick P Rivara, ${ }^{7}$ \\ Ronan A Lyons, ${ }^{2,3}$ Shanthi Ameratunga, ${ }^{8}$ Mariana Brussoni, ${ }^{9,10}$ Fiona E Lecky, ${ }^{11}$ \\ Clare Bradley, ${ }^{12,13}$ Pam M Simpson, ${ }^{1}$ Ben Beck, ${ }^{1}$ Joanne C Demmler, ${ }^{2}$ Jane Lyons, ${ }^{2}$ \\ Amy Schneeberg, ${ }^{10,14}$ James E Harrison ${ }^{15}$
}

To cite: Gabbe BJ, Dipnall JF, Lynch JW, et al. Validating injury burden estimates using population birth cohorts and longitudinal cohort studies of injury outcomes: the VIBESJunior study protocol. BMJ Open 2018;8:e024755. doi:10.1136/ bmjopen-2018-024755

- Prepublication history for this paper is available online. To view these files, please visit the journal online (http://dx.doi org/10.1136/bmjopen-2018024755).

Received 19 June 2018 Revised 28 June 2018 Accepted 6 July 2018
Check for updates

(C) Author(s) (or their employer(s)) 2018. Re-use permitted under CC BY-NC. No commercial re-use. See rights and permissions. Published by BMJ.

For numbered affiliations see end of article.

Correspondence to Dr Belinda J Gabbe; belinda.gabbe@monash.edu

\section{ABSTRACT}

Introduction Traumatic injury is a leading contributor to the global disease burden in children and adolescents, but methods used to estimate burden do not account for differences in patterns of injury and recovery between children and adults. A lack of empirical data on postinjury disability in children has limited capacity to derive valid disability weights and describe the long-term individual and societal impacts of injury in the early part of life. The aim of this study is to establish valid estimates of the burden of nonfatal injury in children and adolescents.

Methods and analysis Five longitudinal studies of paediatric injury survivors $<18$ years at the time of injury (Australia, Canada, UK and USA) and two wholeof-population linked administrative data paediatric studies (Australia and Wales) will be analysed over a 3-year period commencing 2018. Meta-analysis of deidentified patient-level data $(n \approx 2,600)$ from five injury-specific longitudinal studies (Victorian State Trauma Registry; Victorian Orthopaedic Trauma Outcomes Registry; UK Burden of Injury; British Columbia Children's Hospital Longitudinal Injury Outcomes; Children's Health After Injury) and $>1$ million children from two whole-of-population cohorts (South Australian Early Childhood Data Project and Wales Electronic Cohort for Children). Systematic analysis of pooled injury-specific cohort data using a variety of statistical techniques, and parallel analysis of whole-of-population cohorts, will be used to develop estimated disability weights for years lost due to disability, establish appropriate injury classifications and explore factors influencing recovery.

Ethics and dissemination The project was approved by the Monash University Human Research Ethics Committee project number 12311. Results of this study will be submitted for publication in internationally peer-reviewed journals. The findings from this project have the capacity to improve the validity of paediatric injury burden measurements in future local and global burden of disease studies.

\section{Strengths and limitations of this study}

- This is the first study to combine longitudinal cohorts of injured children and adolescents and richly phenotyped whole-of-population linked data studies to provide the best attainable base for obtaining paediatric-specific disability weights and durations of disability.

- The included longitudinal cohort studies of injury outcomes have all achieved low attrition and high follow-up rates.

- This major international collaboration brings together injury experts in the areas of epidemiology, public health, measurement and biostatistics to ensure meaningful and rigorous statistical techniques are applied to the data.

- The primary limitation of this study is that, because equivalent data from low-income and middle-income countries are not yet available, the injury outcome studies included in this study are all from high-income countries.

\section{INTRODUCTION}

Traumatic injury is the most common cause of mortality in adolescents and children beyond 1 year of age and a leading contributor to the global and Australian disease burden in this age group. ${ }^{2}$ While these figures highlight the global importance of injury prevention and trauma care in children and adolescents, the estimates of disease burden are based on methods that have been shown to underestimate the non-fatal burden of injury. ${ }^{34}$ Injury in childhood and adolescence is highly important because of the potential for adverse impacts on a child's prospects for health, education and social inclusion, as well as family and societal impacts. ${ }^{56}$ Detailed and reliable information on the long-term individual and socioeconomic impacts of injury is necessary for effective prioritisation of 
prevention efforts, health and social service planning and to enable accurate assessment of the cost-effectiveness of interventions designed to reduce childhood injury burden.

The 1990 Global Burden of Disease (GBD) study produced comprehensive estimates of the burden of disease, injury and risk factors. ${ }^{78}$ This landmark study, and the subsequent 2010, 2013 and 2015 GBD studies, has been influential in shaping global health priorities. The methodology developed has been widely used in country and region-specific studies, including the recent Australian Burden of Disease Study. ${ }^{2}$ Central to this methodology is a metric for measuring disease burden, called the disability-adjusted life year (DALY). Years of life lost (YLLs) and years lived with disability (YLDs) are combined to calculate DALYs for each disease or condition. The YLL component uses incident mortality data. while calculation of YLDs requires data on the incidence or prevalence of each specific disease or condition, an estimate of the duration of disability and a disability weight for the specific disease or condition. ${ }^{9}$ Despite the widespread use of the DALY metric, weaknesses and uncertainties underscore the need to acquire better data to estimate the disability burden. ${ }^{10} 11$

The GBD estimates of persisting disability after non-fatal injury are particularly problematic as these are based on very limited sources of data. Recent studies, based on follow-up of large cohorts of injured people, have shown that insufficient data underpinned the calculations. ${ }^{3412}$ Several of the assumptions for the methods used for estimating injury burden in the GBD studies were not supported. It has now been shown that longterm individual and socioeconomic impacts are more prevalent after serious injury than is implied by the definition of disability and the disability weights usually used to calculate YLDs. ${ }^{313} 14$ The GBD studies do not use the generally accepted disability definition but a definition of impairment or 'health loss' and their weight sets and parameters for duration and recovery are generally not based on measured outcomes in individual cases. The findings of these recent studies show that current burden estimates underestimate the long-term impacts of most types of serious injury, and important impacts of injury outcomes are not accounted for by the DALY methodology (eg, societal, family, cost and participation).

The GBD disability weights and nature of injury classifications required for calculating DALYs do not take account of the possibility that correct values and distributions for children and adolescents differ from those for adults. Previous studies have applied generic disability weights and nature of injury classifications when estimating childhood injury burden despite dissimilarities in the pattern of injuries and differences in recovery trajectories between children and adults. ${ }^{15-17}$ The types of injuries sustained, and the added complexity of sustaining injuries during key stages of growth and development, create unique challenges to robust estimation of childhood injury burden. ${ }^{9}$
Underlying each GBD disability weight is a short lay description, or vignette, intended to represent in a few words the health impact of a particular type of injury (or disease) on a hypothetical individual. Generally, panel members are shown many pairs of lay descriptions and are asked to decide which of each pair they considered 'healthier than the other'. The vignettes have typically not accounted for differences between children and adults, and the GBD weights have mostly been assumed to be applicable to all ages. ${ }^{3}$ The assumption that generic GBD parameter values are valid for children and adolescents has not been validated.

The paucity of empirical data on postinjury disability in children has contributed to the lack of progress in deriving disability weights and describing the long-term individual and societal impacts of injury in the early part of life ${ }^{61819}$ Existing surveillance systems such as hospital emergency department (ED) presentations, admission datasets and most trauma registries do not include measures of long-term disability and socioeconomic impact and, to date, there have been too few population-based data collections and broader data linkage studies to close this knowledge gap. Most cohort studies of child and adolescent injury have involved relatively small samples of injured children recruited at the child's interface with healthcare, have been limited to short periods of follow-up and restricted to particular types of injury (eg, traumatic brain injury). Furthermore, these injury-specific cohort studies commonly omit cases of injury from certain categories, such as victims of child abuse, self-harm injuries, children with chronic pre-existing conditions and children of non-native language backgrounds, though these groups may well be at high risk for injury occurrence and poor outcome. Their exclusion contributes further to the limitations of data on which to base robust population-based estimates of the burden of childhood injury.

The systematic analysis of existing datasets, increasing access to high-quality primary data and augmenting capacity in the analysis of existing data have been identified as critical components for strengthening health metrics and burden of disease estimates. ${ }^{20}$ The 2012-13 Annual Report of the Australian Government Productivity Commission highlighted the need to harness the power of administrative data for evidence-based analysis to drive better policy outcomes, particularly through more streamlined access and data linkage. ${ }^{21}$ With increased capacity for population-level linked health and social data, and the emergence of large-scale cohort studies of injury outcomes in children and adolescents, the opportunity for focused attention on establishing the burden of child and adolescent injury exists.

The overarching purpose of the project is to establish whether existing metrics for burden of injury based on adult populations are valid for estimating burden of injury in children and adolescents. Specific aims of the project are to: 
1. Characterise and measure deficits in physical and psychosocial functioning and educational attainment following injury in childhood and adolescence.

2. Establish the time-course of recovery following injury in childhood and adolescence.

3. Evaluate the contribution of injury-related characteristics (eg, nature, bodily location, intent and mechanism), factors present at injury (eg, age at injury, developmental history and family characteristics) and factors acting after injury (eg, acute care and rehabilitation), as predictors of recovery following injury in childhood and adolescence.

4. Compare estimates of the burden of injury in childhood and adolescence based on novel metrics from this study with estimates based on existing burden of disease methods, to develop an appropriate model and to determine whether metrics for burden of injury in adulthood provide an adequate basis for estimating burden in children and adolescents.

\section{METHODS AND ANALYSIS}

\section{Study design}

This project is an international collaboration using pooled analysis of five longitudinal studies of injured children and adolescents $(n \approx 2,600)$ and parallel analysis of two whole-of-population, linked data studies $(\mathrm{n} \approx 1.3$ million) in which successive birth cohorts of children in Wales and South Australia have been followed to ascertain injury occurrence and later health, developmental and educational outcomes.

\section{Included datasets}

Aims 1-3 will use data from the five injury-specific longitudinal studies and whole-of-population cohorts. Aim 4 will use the findings from aims 1-3, combined with incidence data from multiple countries. The inclusion of both whole-of-population cohorts and injury-specific longitudinal studies will enable us to compare and contrast findings, providing a comprehensive evaluation of the long-term impact of injury in childhood and adolescence.

All data will be anonymised before provision to the study team and stored securely and only accessible to approved researchers.

\section{Longitudinal cohorts}

Five longitudinal cohort studies of paediatric injury survivors have been selected for this project. The studies were selected as they included all injury types, collected outcomes at multiple time points after injury (including follow-up to at least 12 months after injury), and included standardised measures of function, health status or health-related quality of life instruments. Additional features of the selected studies in this group are the inclusion of injury diagnoses coded using International Classification of Diseases (ICD) or Abbreviated Injury Scale classifications to enable characterisation of the injuries sustained and the presence of external cause of injury codes. Strengths of these studies are the injury-specific focus and primary data collection of patient-centred outcomes at defined time points after injury. The longitudinal injury studies are summarised below and in table 1 .

The Victorian State Trauma Registry (VSTR) is a population-based trauma registry that captures data about all major trauma patients in the state of Victoria (population 5.8 million). ${ }^{22}$ The VSTR uses an opt-off consent process and the opt-off rate is $<0.5 \%$. The registry captures data from the patient's hospital admission including demographic, injury event, injury diagnosis, comorbid status, treatment and inhospital outcomes (ie, mortality, length of stay, discharge destination and so on). Children less than 16 years of age who survive to hospital discharge have been routinely followed-up at 6,12 and 24 months postinjury since March 2010, while older children (16-17 years) have been followed up at these time points since January 2007. The VSTR registers approximately 250 cases per year under the age of 18 years, with an inhospital death rate in this group of around $7 \%$.

In addition, VSTR cases with a date of injury from July 2011 to June 2012 have participated in additional follow-up interviews at 36, 48 and 60 months postinjury through the National Health and Medical Research Council (NHMRC) of Australia funded REcovery after Serious Trauma - Outcomes, Resource use and patient Experiences (RESTORE) study. There are approximately 200 child and adolescent participants in the RESTORE cohort.

Approval has been obtained to link the VSTR and RESTORE study cases with hospitalisation records (ED presentation and admissions), deaths registry data and road traffic injury compensable claims data, focusing on health service utilisation and postdischarge mortality. Once linked, the VSTR and RESTORE dataset will have characteristics similar to the whole-of-population linked datasets.

The Victorian Orthopaedic Trauma Outcomes Registry (VOTOR) is a comprehensive clinical registry of orthopaedic injuries, treatment, complications and outcomes based on admissions to The Alfred, Royal Melbourne, University Hospital Geelong and Northern Hospitals. ${ }^{23}$ Patients are eligible for inclusion if they are over 15 years of age, with an orthopaedic injury diagnosis through the International Classification of Diseases 10th Revision Australian Modification (ICD-10-AM) codes. Patients have been routinely followed up at 6 and 12 months postinjury since 2007, and the 24-month time point was added commencing July 2013. Information on health-related quality of life, function, disability and pain have been collected at these time points.

The UK Burden of Injury (UKBOI) study was designed to measure the population burden of injuries in the UK. The main component was a longitudinal study of injured individuals. Injuries were defined using ICD-10 codes. This prospective study recruited 1517 participants from EDs and hospital inpatients in four UK centres. Participants were recruited between September 2005 and April 2007 and were followed up at 1,4 and 12 months 
Table 1 Summary of participating injury-specific cohort studies

\begin{tabular}{|c|c|c|c|c|c|c|}
\hline Study & Setting & Inclusion criteria & $\begin{array}{l}\text { Participants } \\
<18 \text { years }\end{array}$ & $\begin{array}{l}\text { Follow-up } \\
\text { time points }\end{array}$ & $\begin{array}{l}\text { Key outcome } \\
\text { measures }\end{array}$ & $\begin{array}{l}\text { Injury diagnosis } \\
\text { coding }\end{array}$ \\
\hline VSTR & Australia & $\begin{array}{l}\text { Inhospital death, ISS }>12 \text {, ICU } \\
\text { admission or urgent surgery, } \\
\text { met burns criteria } 20 \%- \\
29 \% \text { full/partial thickness. }\end{array}$ & $n=2204^{*}$ & $\begin{array}{l}6,12 \text { and } \\
24 \text { months }\end{array}$ & $\begin{array}{l}\text { EQ-5D-3L, PedsQL, } \\
\text { GOSE, KOSCHI and } \\
\text { WHODAS. }\end{array}$ & $\begin{array}{l}\text { ICD-10-AM and AIS } \\
2005 \text { (2008 Update). }\end{array}$ \\
\hline VOTOR & Australia & $\begin{array}{l}\text { Orthopaedic injury } \\
\text { admission }>24 \text { hours or death } \\
\text { within } 24 \text { hours. }\end{array}$ & $\mathrm{n}=1041^{*}$ & & $\begin{array}{l}\text { EQ-5D-3L, } \\
\text { GOSE and } \\
\text { WHODAS. }\end{array}$ & ICD-10-AM. \\
\hline $\mathrm{CHAl}$ & USA & $\begin{array}{l}\text { Presentation to ED or hospital } \\
\text { admission for either a TBI or an } \\
\text { upper extremity injury. }\end{array}$ & $n=926$ & $\begin{array}{l}3,12, \\
24 \text { and } \\
36 \text { months }\end{array}$ & $\begin{array}{l}\text { PedsQL, ABAS- } \\
\text { II and CASP. }\end{array}$ & ICD-9-CM. \\
\hline UKBOI & UK & $\begin{array}{l}\text { Presentation to ED or hospital } \\
\text { admission } \\
5+\text { years. }\end{array}$ & $n=181$ & $\begin{array}{l}1,4 \text { and } \\
12 \text { months }\end{array}$ & $\begin{array}{l}\text { EQ-5D or } \\
\text { PedsQL and HUI3. }\end{array}$ & $\begin{array}{l}\text { ICD-10 and } \\
\text { AIS2005. }\end{array}$ \\
\hline
\end{tabular}

*A proportion of patients met VSTR and VOTOR inclusion criteria $(n=273)$, and duplicated information will be omitted from VOTOR data. ABAS-II, Adaptive Behavior Assessment System-Second Edition; AIS, Abbreviated Injury Scale; BCCH-LIO, British Columbia Children's Hospital Longitudinal Injury Outcomes study; CASP, Child and Adolescent Scale of Participation; CHAI, Children's Health After Injury; CHIRRP, Canadian Hospital Injury Reporting and Prevention Program; ED, emergency department; GOSE, Glasgow Outcome Scale - Extended; HUI3, Health Utilities Index 3; ICD, International Classification of Diseases; ICU, intensive care unit; ISS, Injury Severity Score; KOSCHI, King's Outcome Scale for Closed Head Injury; PedsCTAS, Paediatriac Canadian Triage and Acuity Scale; PedsQL, Pediatric Quality of Life Inventory; TBI, traumatic brain injury; UKBOI, UK Burden of Injury study; VSTR, Victorian State Trauma Registry; VOTOR, Victorian Orthopaedic Trauma Outcomes Registry; WHODAS, World Health Organisation Disability Assessment Scale.

postinjury by postal questionnaire. Twelve per cent of participants were under the age of 18 years at the time of injury.

The Children's Health After Injury (CHAI) study was a multicentre, collaborative effort between researchers at the University of Washington School of Medicine, Seattle Children's Hospital and The Children's Hospital of Philadelphia. The CHAI study recruited 729 children with mild, moderate and severe traumatic brain injury and 197 children with upper extremity injuries between March 2007 and September 2008. These participants were followed up at 3,12, 24 and 36 months after injury using online, telephone and postal questionnaires.

The British Columbia Children's Hospital Longitudinal Injury Outcomes (BCCH-LIO) study is a prospective cohort study of 351 children aged $0-16$ years who attended the British Columbia Children's Hospital for an injury between February 2011 and December 2013. ${ }^{19} 24$ Surveys assessed health-related quality of life at four time points: baseline (preinjury), 1, 4 and 12 months postinjury by postal questionnaire. The BCCH-LIO study cases will be linked to hospital admissions and general practice consultations through PopData BC and to public drug insurance programmes for eligible, low-income participants, prior to the provision of data for the paediatric Validating Injury Burden Estimates Study (VIBES-Junior).

Whole-of-population linked administrative data studies

The whole-of-population linked administrative data studies access information on all children born or living in a specified region or country over a defined timeframe. For this project, these studies use anonymised linked data that include births and deaths, maternal and child health, hospital admissions, ED data and a range of developmental and educational outcomes. The key strengths of using administrative data cohorts are the ability to capture data from the whole population, including subgroups of the population who are often excluded, or choose not to participate in studies requiring participant consent (eg, cases due to family violence and in disadvantaged groups), and linkage to a range of non-health related datasets that may contribute a greater diversity of risk factor and/or outcome measurement. A strength of this study is the opportunity to explore injury outcomes relative to comparison groups of healthy children or those experiencing other health conditions. The whole-of-population linked cohorts to be included are summarised here.

The South Australia Early Childhood Data Project contains the complete birth cohorts of children born in South Australia from 1991 to 2016. These birth cohorts include approximately 500000 children of whom 18000 are Aboriginal. Many different government data sources contribute over 10 million records. Data include pregnancy and birth, birth defects, community maternal and child health services, child development, preschool, educational attainment, behavioural problems and school attendance, child maltreatment, and hospitalisation and emergency department data. 
The Welsh Electronic Cohort for Children is a total population anonymised electronic cohort study of all children ( $\mathrm{n}=1254433$ ) living in Wales and born between 1990 and 2016, set up using the Secure Anonymised Information Linkage (SAIL) databank. ${ }^{25-29}$ Eligible participants were identified from the Welsh Demographic Service (NHS Register), and the National Community and Child Health Database will be used for validation in this study. Individual-level anonymised data on these children are obtained from the following datasets: SAIL General Practice Data; Patient Episode Database for Wales for inpatient and outpatient episodes (1996-); Emergency Department Data Set (2009-); Critical Care Minimum Dataset (2006-); the National Pupil Database; and the Pupil Level Annual School Census. Approval has been obtained to include data from the Trauma Audit and Research Network, the trauma registry for England and Wales, which will enable further detailed characterisation of 1929 seriously injured children for VIBES-Junior.

\section{Data analysis}

The distinct characteristics of the two types of data require different management and analytic techniques.

\section{Injury-specific cohort studies}

Anonymised participant-level study data will be provided by the data custodians. The injury-specific cohort datasets will be harmonised and pooled for analysis, as was undertaken in the original Injury-VIBES project. ${ }^{3} 30$ The range of diagnosis codes, and combinations of diagnosis codes, across the datasets will be summarised. Diagnostic codes will be mapped to the most recent GBD study injury health states and European Injury Database Groupings ${ }^{331}$ and will be reported in terms of ICD-10 categories where case numbers are sufficient. The relevance of existing classifications to the child and adolescent injury population will be explored, and alternative injury health state classifications will be developed for testing and evaluation, where discrepancies arise.

The calculation of disability weights for YLD estimates from empirical data requires the use of multiattribute utility instruments (MAUIs). The EQ-5D-3L (EQ-5D) is a generic measure of health status, consisting of a descriptive component asking about problems in five dimensions (mobility, self-care, usual activities, pain or discomfort and anxiety or depression) and the most widely used MAUI for this purpose. ${ }^{32}$ The EQ-5D is captured by the VSTR, VOTOR, UKBOI and BCCH-LIO studies (table 1). The Pediatric Quality of Life inventory (PedsQL) is a 23-item generic health-related quality of life instrument designed to measure the dimensions of physical, mental, social health and role function (school) in children. ${ }^{33}$ Responses to each of the items are used to generate physical and psychosocial health summary scores, ranging from 0 to 100 with higher scores representing better function. The VSTR, VOTOR, UKBOI, CHAI and BCCH-LIO used this instrument to measure paediatric injury outcomes. The PedsQL is not a MAUI, but a published map of this instrument to the EQ-5D will be used, ${ }^{34}$ and the map validated using the participating datasets that collect both measures.

Summary statistics will be used to describe the profile of injury cases in each dataset, including the age at time of injury, sex, comorbid status, socioeconomic profile, cause of injury and other key population descriptors. The prevalence of problems at each follow-up time point will be assessed, and data visualisation techniques will be used to show injury outcomes over time and by key patient subgroups. Multilevel random effects regression modelling will be used to assess the rate of change in outcomes over time. Using these models, the probability of reporting persistent problems at each follow-up time point will be established and compared between injury groups, and these analyses will be used to establish the duration of persisting problems for the calculation of YLDs.

Where linked administrative data are available for the cohorts (UKBOI, VSTR, VOTOR and BCCH-LIO), the pattern of healthcare service utilisation will be quantified. Episodes of care related to the index injury event will be separated from new injury events (ie, injury recidivism) and contact with health services for other conditions.

The whole-of-population linked record studies do not include MAUIs, but they do include other data that might indicate health status, notably data on health service utilisation. Where both MAUIs and health service utilisation data are available in the injury-specific cohorts, associations will be analysed as a basis for strengthening interpretation of findings based on the whole-of-population linked administrative data studies.

\section{Whole-of-population linked administrative data studies}

Parallel analysis of the whole-of-population cohorts will be undertaken, following harmonisation of data items and agreed analysis plans. Summary statistics will be used to describe the profile of injury cases in each dataset, relative to participants without injury, including the age, sex, comorbid status, socioeconomic profile, cause of injury and other key population descriptors. The rates of hospitalisation in childhood for injury overall, and by type of injury, will be established and compared with the rates of hospitalisation for other conditions. Injury recidivism will be investigated using survival analyses for recurrent events. Characteristics in the period under observation but before occurrence of the reference injury event period, such as health service utilisation profiles, and in an equivalent prebaseline period for non-injured comparison groups, will be analysed as potential predictors of postinjury health state.

The pattern of healthcare service utilisation will be quantified, and the findings will be compared with the analyses based on the injury-specific cohort studies. Episodes of care related to the cohort injury event will be separated from new injury events (ie, injury recidivism) and contact with health services for other conditions. Educational outcomes at key assessment stages will 
be compared between injury groups, with an uninjured comparison group, and over time, to assess the impact of injury on educational attainment. A variety of regression approaches appropriate for the data will be used from the family of generalised linear models, accounting for confounding. The linked data cohorts have extensive data on socioeconomic, perinatal, family and background risk. These will be analysed as potential risk factors for injury occurrence and as factors that may influence recovery where injury has occurred.

\section{Application of novel YLD metrics to incident data}

The MAUI data from the injury-specific cohorts will be used to calculate paediatric-specific disability weights for common injury diagnoses and the categories in key injury classifications. These will be compared with existing panel-derived weight sets and with the novel weight sets from the Injury-VIBES study generated using adult injury outcome datasets. ${ }^{3}$

To establish the impact of paediatric-specific disability weights and durations of disability on YLD estimates, population-level incidence data are required. For each population dataset, the principal diagnosis for the injury admission will be mapped to the applicable GBD injury classification. YLD estimates will then be calculated using:

i. The novel, paediatric-specific disability weights and durations of disability established from the current study.

ii. The novel, adult-specific disability weights and durations of disability from the original Injury-VIBES project. $^{3}$

iii. The applicable GBD injury disability weights and durations.

To achieve this, we will collaborate with the Australian Institute of Health and Welfare's Australian Burden of Disease investigators, and with researchers at the University of British Columbia, to apply the findings to Australian and Canadian hospitalisations data, respectively. In the USA, data will be obtained from the Healthcare Cost and Utilization Project to allow the research team to directly apply the findings to US hospitalisations data. The UKBOI team managed data from 22 countries participating in the European Injury Database and has developed a web-based YLD and DALY tool to support individual countries in measuring injury burden using disability weights from the original Injury-VIBES study. ${ }^{35}$ This tool will be used to apply the findings of the current study. Where possible, the extent to which social deprivation impacts on the YLD estimates will be reviewed to infer the global application in low-income and middle-income countries.

\section{Patient and public involvement}

Existing datasets will be used for the purpose of this study. Patient and public involvement was not used in the design of this study. The extent to which patients and the public were involved in the planning and design of the contributing studies can be sought from the published papers and websites of the participating studies.

\section{Ethics and dissemination}

The project will be conducted in compliance the NHMRC National Statement on ethical Conduct in Human Research (2007) and the Note for Guidance on Good Clinical Practice (CPMP/ICH-135/95). Results of this study will be submitted for publication in internationally peer-reviewed journals. The data included in this project have been provided by the original data custodians specifically for this project and are not freely available. Requests for access to data from the participating datasets would need to be directed to the relevant data custodian.

Prevention of deaths due to injury remains important, but increased attention to the morbidity and other outcomes is needed due to the greater incidence of non-fatal cases of serious injury. In contrast to GBD findings, the burden on society of disability due to non-fatal injury has been found to substantially outweigh the burden of mortality, when it is measured well. ${ }^{4}$ The analyses proposed here will focus, for the first time, on developing burden metrics specifically for child and adolescent injury populations based on the best available data. This will provide evidence-informed metrics to improve burden estimates. The findings of the proposed project will have significant benefits for understanding the burden of injury internationally, providing essential data and inputs for prioritisation of prevention efforts, health and social service planning, and the capacity to determine the cost-effectiveness of interventions designed to reduce childhood injury burden. Learnings from this study are likely to be applicable to other countries and to other health conditions. The findings are likely to influence how injury burden is measured in future GBD studies and in other country-specific or regional burden studies.

\section{Author affiliations}

${ }^{1}$ School of Public Health and Preventive Medicine, Monash University, Melbourne, Victoria, Australia

${ }^{2}$ Health Data Research UK, Swansea University, Swansea, UK

${ }^{3}$ National Centre for Population Health and Wellbeing Research, Swansea University,

Swansea, UK

${ }^{4}$ School of Medicine, Deakin University, Geelong, Victoria, Australia

${ }^{5}$ School of Public Health, University of Adelaide, Adelaide, South Australia, Australia

${ }^{6}$ School of Population Health Sciences, University of Bristol, Bristol, UK

${ }^{7}$ Departments of Pediatrics and Epidemiology, and the Harborview Injury

Prevention and Research Center, University of Washington, Seattle, Washington, USA

${ }^{8}$ School of Population Health, University of Auckland, Auckland, New Zealand ${ }^{9}$ Department of Pediatrics, School of Population and Public Health, University of British Columbia, Vancouver, Canada

${ }^{10}$ British Columbia Injury Research and Prevention Unit, Children's Hospital

Research Institute, Vancouver, Canada

${ }^{11}$ School of Health and Related Research, University of Sheffield, Sheffield, UK

${ }^{12}$ South Australian Health and Medical Research Institute, Adelaide, South Australia, Australia

${ }^{13}$ School of Medicine, Flinders University, Adelaide, South Australia, Australia

${ }^{14}$ School of Population and Public Health, University of British Columbia, Vancouver, Canada

${ }^{15}$ Research Centre for Injury Studies, Flinders University, Adelaide, South Australia, Australia 
Acknowledgements The authors would like to extend their gratitude and acknowledgements to all study participants and study team members for their time and energy spent on this project.

Contributors All authors are investigators of the project, contributed to the study design and funding application. BJG drafted the manuscript. JFD, JL, FPR, RAL, SA, $\mathrm{MB}, \mathrm{FEL}, \mathrm{CB}, \mathrm{PMS}, \mathrm{BB}, \mathrm{JCD}, \mathrm{JL}, \mathrm{AS}$ and JEH reviewed the manuscript and approved the final version of the manuscript.

Funding The VIBES-Junior project is funded by the National Health and Medical Research Council of Australia project grant (APP1142325). The UKBOI study was funded by the Policy Research Programme in the Department of Health (Grant number: 0010009). The VSTR is a Department of Health (Victoria) and Transport Accident Commission (TAC) funded initiative. VOTOR is funded by the TAC through the Institute for Safety, Compensation and Recovery Research. The SAECDP is funded by NHMRC Partnership Project Grant (1056888), NHMRC Centre of Research Excellence (1099422) and the Government of South Australia. The CHAl project was funded by a grant from the US Centers for Disease Control and Prevention. WECC is funded through Health and Care Research Wales and Health Data Research UK. The BCCH-LIO was funded by the Canadian Institutes of Health Research (Grant \#TIR104028) and the Michael Smith Foundation for Health Research. Salary support for MB was provided by the BCCH Research Institute and by a Michael Smith Foundation for Health Research Scholar Award. BJG was supported by an Australian Research Council Future Fellowship (FT170100048).

Competing interests None declared.

Patient consent Not required.

Ethics approval The project was approved by the Monash University Human Research Ethics Committee project number 12311.

Provenance and peer review Not commissioned; peer reviewed for ethical and funding approval prior to submission.

Open access This is an open access article distributed in accordance with the Creative Commons Attribution Non Commercial (CC BY-NC 4.0) license, which permits others to distribute, remix, adapt, build upon this work non-commercially, and license their derivative works on different terms, provided the original work is properly cited, appropriate credit is given, any changes made indicated, and the use is non-commercial. See: http://creativecommons.org/licenses/by-nc/4.0/.

\section{REFERENCES}

1. Kyu HH, Pinho C, Wagner JA, et al. Global and national burden of diseases and injuries among children and adolescents between 1990 and 2013: findings from the global burden of disease 2013 study. JAMA Pediatr 2016;170:267-87.

2. Hoy WE. Australian burden of disease study: impact and causes of illness and death in Australia 2011. Australian burden of disease study series. Canberra: Australian Instittue of Health and Welfare, 2016.

3. Gabbe BJ, Lyons RA, Simpson PM, et al. Disability weights based on patient-reported data from a multinational injury cohort. Bull World Health Organ 2016;94:806-16.

4. Lyons RA, Kendrick D, Towner EM, et al. Measuring the population burden of injuries-implications for global and national estimates: a multi-centre prospective UK longitudinal study. PLOS Med 2011;8:e1001140.

5. Peden M, Oyegbite K, Ozanne-Smith J, et al. In: Organization WH, ed. World report on child injury prevention. Geneva, Switzerland: World Health Organization, 2008.

6. Rivara FP, Oldham KT. Pediatric trauma care: defining a research agenda. J Trauma 2007;63:S52-S53.

7. Lopez A, Mathers C, Ezzati M, et al. Global burden of disease and risk factors. New York: Oxford University Press, 2006.

8. Horton R. GBD 2010: understanding disease, injury, and risk. Lancet 2012;380:2053-4.

9. Polinder S, Haagsma JA, Lyons RA, et al. Measuring the population burden of fatal and nonfatal injury. Epidemiol Rev 2012;34:17-31.

10. Reidpath DD, Allotey PA, Kouame A, et al. Measuring health in a vacuum: examining the disability weight of the DALY. Health Policy Plan 2003;18:351-6.

11. Voigt K, King NB. Disability weights in the global burden of disease 2010 study: two steps forward, one step back? Bull World Health Organ 2014;92:226-8.
12. Gabbe BJ, Simpson PM, Lyons RA, et al. Association between the number of injuries sustained and 12-month disability outcomes: evidence from the injury-VIBES study. PLoS One 2014;9:e113467.

13. Derrett $S$, Wilson $S$, Samaranayaka A, et al. Prevalence and predictors of disability 24-months after injury for hospitalised and non-hospitalised participants: results from a longitudinal cohort study in New Zealand. PLoS One 2013;8:e80194.

14. Gabbe BJ, Simpson PM, Harrison JE, et al. Return to work and functional outcomes after major trauma: who recovers, when, and how well? Ann Surg 2016;263:623-32.

15. Murray CJ, Vos T, Lozano R, et al. Disability-adjusted life years (DALYs) for 291 diseases and injuries in 21 regions, 1990-2010: a systematic analysis for the Global Burden of Disease Study 2010. Lancet 2012;380:2197-223.

16. Murray CJL, Barber RM, Foreman KJ, et al. Global, regional, and national disability-adjusted life years (DALYs) for 306 diseases and injuries and healthy life expectancy (HALE) for 188 countries, 1990-2013: quantifying the epidemiological transition. The Lancet 2015;386:2145-91.

17. Murray CJ, Barber RM, Foreman KJ, et al. Global, regional, and national disability-adjusted life years (DALYs) for 306 diseases and injuries and healthy life expectancy (HALE) for 188 countries, 1990-2013: quantifying the epidemiological transition. Lancet 2015;386:2145-91.

18. Gabbe BJ, Simpson PM, Sutherland AM, et al. Functional and health-related quality of life outcomes after pediatric trauma. $J$ Trauma 2011;70:1532-8.

19. Schneeberg A, Ishikawa T, Kruse S, et al. A longitudinal study on quality of life after injury in children. Health Qual Life Outcomes 2016;14.

20. Murray CJ, Frenk J. Health metrics and evaluation: strengthening the science. Lancet 2008;371:1191-9.

21. Productivity Commission. Annual report 2012-13. Annual Report Series. Canberra: Productivity Commission, 2013.

22. Cameron PA, Finch CF, Gabbe BJ, et al. Developing Australia's first statewide trauma registry: what are the lessons? ANZ J Surg 2004;74:424-8.

23. Edwards E, Graves S, McNeil J, et al. Orthopaedic trauma: establishment of an outcomes registry to evaluate and monitor treatment effectiveness: Elsevier, 2006.

24. Bell N, Kruse S, Simons RK, et al. A spatial analysis of functional outcomes and quality of life outcomes after pediatric injury. Inj Epidemiol 2014;1.

25. Hutchings HA, Evans A, Barnes $P$, et al. Do children who move home and school frequently have poorer educational outcomes in their early years at school? An anonymised cohort study. PLoS One 2013;8:e70601.

26. Hyatt M, Rodgers SE, Paranjothy S, et al. The wales electronic cohort for children (WECC) study. Arch Dis Child Fetal Neonatal Ed 2011;96:Fa18.

27. Lyons R, Hutchings $\mathrm{H}$, Rodgers $\mathrm{S}$, et al. Development and use of a privacy-protecting total population record linkage system to support observational, interventional, and policy relevant research. Lancet 2012.

28. Lyons RA, Jones $\mathrm{KH}$, John $\mathrm{G}$, et al. The SAIL databank: linking multiple health and social care datasets. BMC Med Inform Decis Mak 2009;9:3.

29. Gabbe BJ, Brooks C, Demmler JC, et al. The association between hospitalisation for childhood head injury and academic performance: evidence from a population e-cohort study. J Epidemiol Community Health 2014;68:466-70.

30. Lyons RA, Gabbe BJ, Simpson PM, et al. 47 Disability weights from the injury vibes collaboration: BMJ Publishing Group Ltd, 2016.

31. Haagsma JA, Graetz N, Bolliger I, et al. The global burden of injury: incidence, mortality, disability-adjusted life years and time trends from the Global Burden of Disease study 2013. Inj Prev 2016;22:3-18.

32. Haagsma JA, Polinder S, Cassini A, et al. Review of disability weight studies: comparison of methodological choices and values. Popul Health Metr 2014;12.

33. Varni JW, Seid M, Rode CA. The PedsQL: measurement model for the pediatric quality of life inventory. Med Care 1999;37:126-39.

34. Khan KA, Petrou S, Rivero-Arias O, et al. Mapping EQ-5D utility scores from the PedsQL ${ }^{\mathrm{TM}}$ generic core scales. Pharmacoeconomics 2014;32:693-706.

35. Turner S, Lyons R, Rogmans W, et al. The European Injury Database: supporting injury prevention and research across Europe. Injury Prevention 2016;22:A80. 